\title{
Management of Spontaneous Subarachnoid Hemorrhage Patients with Negative Initial Digital Subtraction Angiogram Findings: Conservative or Aggressive?
}

\author{
Liang Xu, ${ }^{1,2,3}$ Yuanjian Fang, ${ }^{1,2,3}$ Xudan Shi, ${ }^{4}$ Xianyi Chen, ${ }^{1,2,3}$ Jun Yu, ${ }^{1,2,3}$ Zeyu Sun, ${ }^{1,2,3}$ \\ Jianmin Zhang, ${ }^{1,2,3}$ and Jing $\mathrm{Xu}^{1,2,3}$ \\ ${ }^{1}$ Department of Neurosurgery, The Second Affiliated Hospital, School of Medicine, Zhejiang University, Hangzhou, Zhejiang, China \\ ${ }^{2}$ Brain Research Institute, Zhejiang University, Hangzhou, Zhejiang, China \\ ${ }^{3}$ Collaborative Innovation Center for Brain Science, Zhejiang University, Hangzhou, Zhejiang, China \\ ${ }^{4}$ Department of Anesthesiology, The Second Affiliated Hospital, School of Medicine, Zhejiang University, Zhejiang, China
}

Correspondence should be addressed to Jing Xu; jingxu@zju.edu.cn

Received 24 January 2017; Accepted 21 March 2017; Published 2 May 2017

Academic Editor: Gang Chen

Copyright (C) 2017 Liang Xu et al. This is an open access article distributed under the Creative Commons Attribution License, which permits unrestricted use, distribution, and reproduction in any medium, provided the original work is properly cited.

\begin{abstract}
Background. The ideal management of SAH patients with negative initial DSA findings remains unresolved. Objective. (i) To present risk factors, clinical courses, and outcomes in different types of SAH patients with negative DSA findings; (ii) to explore the differences of basal vein between aSAH patients and NASAH patients; and (iii) to evaluate the value of repeated DSA for these patients. Methods. All SAH patients with negative initial DSA findings between 2013 and 2015 in our hospital were enrolled and were further categorized as perimesencephalic SAH (PMN-SAH) or nonperimesencephalic SAH (nPMN-SAH). Risk factors, clinical courses, outcomes, and the basal vein drainage patterns were compared. Results. A total of 137 patients were enrolled in the present study. The PMN-SAH group had better GOS and mRS values at 1-year follow-up. Moreover, the nPMN-SAH group had a higher rate of complications. The basal vein drainage pattern showed significant difference when comparing each of the NASAH subtypes with aSAH groups. There was a significant higher rate of a responsible aneurysm in nPMN-SAH group upon repeated DSA. Conclusions. SAH patients with negative initial DSA findings had benign clinical courses and outcomes. Repeated DSA studies are strongly advised for patients with the nPMN-SAH pattern.
\end{abstract}

\section{Introduction}

Spontaneous subarachnoid hemorrhage (SAH), characterized by bleeding into the subarachnoid space in the absence of trauma, is most often caused by the rupture of an intracranial aneurysm $[1,2]$. However, according to the previous studies $[3,4]$, even though there is now widespread use of digital subtraction angiogram (DSA) to aid in the diagnosis of spontaneous SAH, nearly fifteen percent of cases remain idiopathic [4]. This category of SAH has been termed nonaneurysmal SAH (NASAH), which typically follows a benign clinical course and has a generally favorable prognosis when compared with aneurysmal subarachnoid hemorrhage (aSAH) $[5,6]$.
According to the distribution pattern of the subarachnoid blood, these NASAH patients are usually divided into two subcategories, perimesencephalic (PMN-SAH) and nonperimesencephalic hemorrhage (nPMN-SAH) [7]. Although it is generally recognized that NASAH has a more preferable outcome than aSAH [5], recent studies suggest that the management of the nPMN-SAH subgroup should be more rigorous in light of its more severe clinical courses and outcomes $[8,9]$. As nPMN-SAH is a diagnosis of exclusion, some controversial issues remain regarding the management of these patients that have negative initial DSA findings $[10,11]$. Despite numerous studies utilizing multiple imaging modalities, the bleeding source of NASAH has not been elucidated. Though most authors support the hypothesis that 
the culprit of the bleeding is of venous origin, the precise mechanism of the bleeding source remains unknown [12]. Furthermore, given the low detection rate of a responsible intracranial lesion in NASAH patients, the need for serial DSAs in these cases remains an open question.

Here we retrospectively analyzed the data of all $\mathrm{SAH}$ patients with initial negative DSA findings in our center between 2013 and 2015. The risk factors, clinical courses, and outcomes of these patients were evaluated in our study. In addition, the venous drainage patterns in both subgroups were compared. Finally, the necessity of repeated DSA examinations was also evaluated based on our study and the review of related literature.

\section{Methods}

2.1. Patients and Procedure. The data of patients who presented with SAH between 2013 and 2015 in our center (Second Affiliated Hospital, School of Medicine, Zhejiang University) were retrospectively analyzed. According to standard management of SAH patients $[13,14]$, all patients were screened by CTA upon admission, followed by emergent DSA examination. There were 164 SAH patients who had an initial CTA that failed to definitively demonstrate a culprit lesion. Of these, 13 were excluded due to a history of traumatic brain injury or another definitive cause of SAH, leaving 151 patients with negative CTA findings. Among them, 14 patients had positive initial DSA results (2 with a perimesencephalic pattern and 12 with a nonperimesencephalic pattern). This left 137 SAH patients with negative initial DSA findings (hereafter referred to as NASAH patients) who were enrolled in the present study. Based on the distribution of the subarachnoid blood, NASAH patients were divided into PMN-SAH ( $n=82$ patients) and nPMN-SAH groups $(n=$ 55) [15]. To further exclude intracranial aneurysm as the source of hemorrhage [16], all of them underwent a repeated DSA examination either 10-14 days after admission or one month after discharge. In total, 57 patients agreed to undergo a repeated DSA during hospitalization or follow-up. Four patients were found to have a culprit intracranial aneurysm on the second angiogram, all of whom were patients with the nonperimesencephalic pattern (Figure 1).

The demographic data includes patient sex, age, smoking history, alcohol use, hypertension, diabetes, and history of anticoagulant use. The Glasgow Coma Score (GCS), HuntHess $(\mathrm{HH})$ grade, and the modified Fisher Scale (mFS) were used for evaluation upon admission [5,33]. The length of hospital stay (LOS) and in-hospital complications, namely, hydrocephalus, cerebral vasospasm, and rebleeding, were compared between the two groups. All patients were followed up by telephone interview or outpatient clinic at three months and one year after discharge. The outcomes were evaluated using the modified Rankin Scale (mRS) and Glasgow Outcome Scale (GOS).

In order to find a potential venous source of bleeding, we compared the basal vein of Rosenthal (BVR) anatomy among the different groups. Excluding 4 patients who were later found to have a definite bleeding source, the venous configurations of 133 NASAH patients (82 PMN-SAH and $51 \mathrm{nPMN}-\mathrm{SAH}$ ) were evaluated and compared to a total of 133 consecutive aSAH patients during the same period. The classification of the BVR was performed as described by Ramazan Buyukkaya et al. [12]. Briefly, the drainage pattern of unilateral BVR was divided into three types:

(1) Type A (normal continuous): a continuous BVR drains mainly into the vein of Galen.

(2) Type B (normal discontinuous): in a discontinuous BVR, the anterior part drains into the sphenoparietal sinus or cavernous sinus through an uncal vein and posterior part drains to the vein of Galen.

(3) Type C (primitive variant): this variant drains mainly to the dural sinuses, not via the vein of Galen (i.e., the perimesencephalic veins drain into the superior petrosal sinus or the BVR drains directly into the transverse or straight sinus).

2.2. Statistical Analysis. Categorical variables of different groups, such as the parameter of demographic data, GCS, $\mathrm{HH}$ grade, $\mathrm{mFS}, \mathrm{mRS}$, and GOS, were compared using the Chi-squared test or Fisher's exact test. Continuous variables, such as the number of patients in each group, were compared using Student's $t$-test. $p<0.05$ was considered as significant statistical difference.

\section{Results}

3.1. Demographics. The demographics of all NASAH patients with negative initial DSA findings are summarized in Table 1. There were no significant differences in sex, age, smoking history, alcohol abusing history, diabetes, hypertension, or anticoagulation medication history between PMN-SAH and nPMN-SAH groups ( $p>0.05$ for each parameter).

3.2. Clinical Course and Risk Factors for Hydrocephalus. The patients' GCS, HH scale, and mFS were evaluated upon arrival at our emergency room. Despite the interval from ictus to evaluation, the nPMN-SAH group presented with higher GCS and mFS on their initial CT scan $(p<0.05)$. However, there was no significant difference in the $\mathrm{HH}$ grade between the two groups ( $p=0.157$ ) (Table 2).

Complications that developed during hospitalization, hydrocephalus, cerebral vasospasm, and rebleeding were compared. The nPMN-SAH group was associated with higher incidence of hydrocephalus and symptomatic cerebral vasospasm ( $p=0.001$ and 0.017 , resp.). Only one patient experienced rebleeding but no definite source of bleeding was confirmed even though a repeat DSA was conducted. There was no significant difference in the rebleeding rate during hospitalization between the two groups $(p=0.401)$ (Table 2).

There were 14 patients (10.2\%) who developed hydrocephalus. The risk factors for hydrocephalus in these patients were compared. There were significant differences in the GCS and mFS between the hydrocephalus group and nonhydrocephalus group $(p<0.001)$. Furthermore, patients who developed hydrocephalus were associated with significantly 


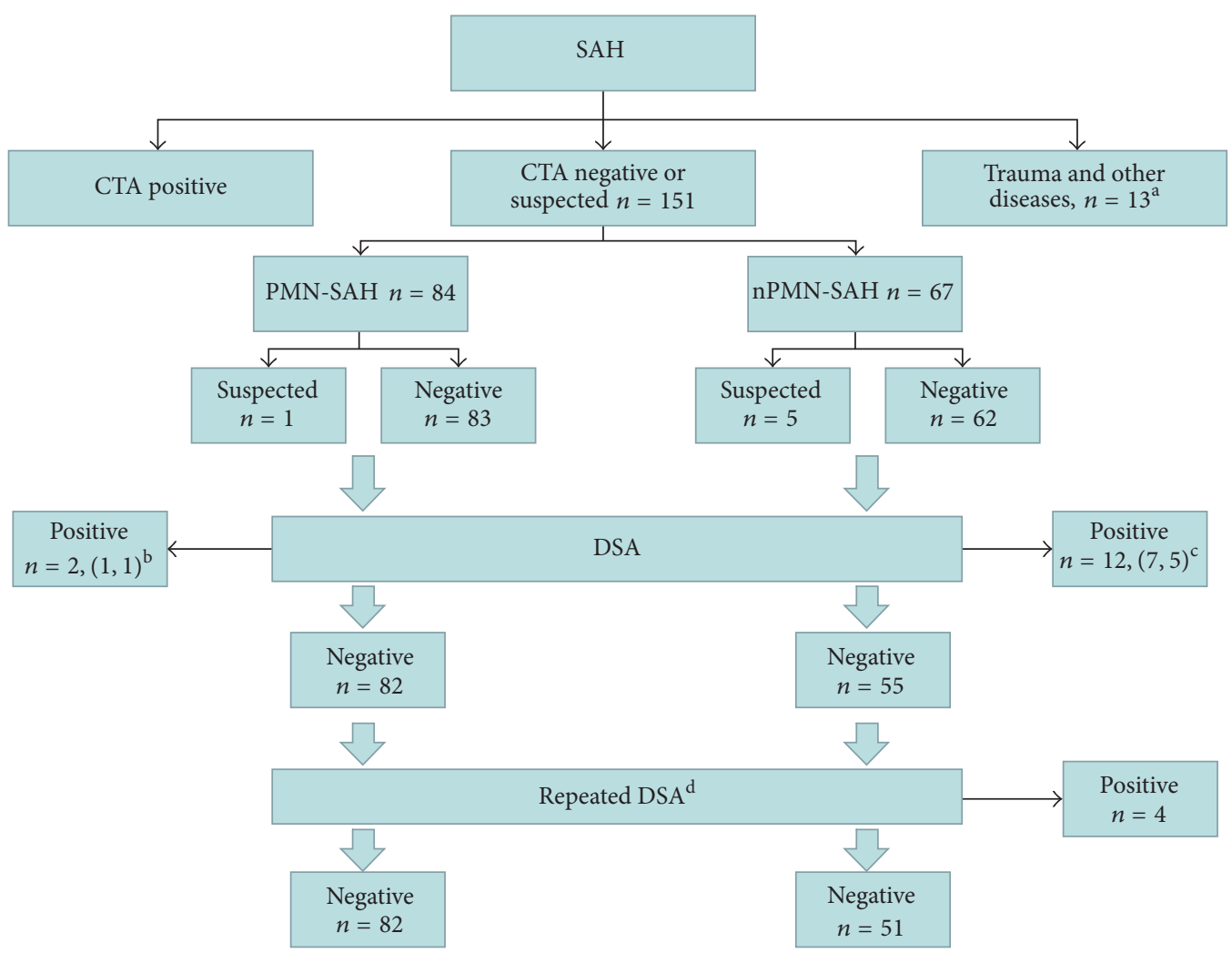

a: 13 patients were excluded who were confirmed with head trauma, Moyamoya disease, venous sinus thrombosis, and other diseases. b: Initial DSA revealed a responsible aneurysm in 2 PMN-SAH patients, 1 of them was found by the initial CTA. c: Initial DSA revealed a responsible aneurysm in $7 \mathrm{nPMN}-\mathrm{SAH}$ patients, 5 of them was found by the initial CTA d: On repeat evaluation, $4 \mathrm{nPMN}-\mathrm{SAH}$ were diagnosed with a responsible intracranial aneurysm.

FIGURE 1

TABLE 1: Demographics of patients with nonaneurysmal subarachnoid hemorrhage.

\begin{tabular}{|c|c|c|c|c|}
\hline & NASAH & PMN-SAH & nPMN-SAH & $p$ value \\
\hline Sum (\%) & $137(100 \%)$ & $82(59.9 \%)$ & $55(40.1 \%)$ & NS \\
\hline Male-female ratio & $76 / 61(1.2 / 1)$ & $42 / 40(1.05 / 1)$ & $33 / 22(1.5 / 1)$ & 0.357 \\
\hline Age (range) & $56.0 \pm 10.4(30-80)$ & $55.4 \pm 9.6(34-76)$ & $56.7 \pm 13.7(30-80)$ & 0.156 \\
\hline Smoker (\%) & $18(13.1 \%)$ & $11(13.4 \%)$ & $7(12.7 \%)$ & 0.907 \\
\hline Alcohol abusing (\%) & $21(15.3 \%)$ & $13(15.9 \%)$ & $8(14.5 \%)$ & 0.835 \\
\hline Diabetes (\%) & $7(5.1 \%)$ & $3(3.7 \%)$ & $4(7.3 \%)$ & 0.438 \\
\hline Hypertension (\%) & $30(21.9 \%)$ & $15(18.3 \%)$ & $15(27.3 \%)$ & 0.213 \\
\hline Anticoagulant Using (\%) & $4(2.9 \%)$ & $2(2.4 \%)$ & $2(3.6 \%)$ & 1 \\
\hline
\end{tabular}

NASAH: nonaneurysmal subarachnoid hemorrhage, PMN-SAH: perimesencephalic nonaneurysmal subarachnoid hemorrhage, nPMN-SAH: nonperimesencephalic nonaneurysmal subarachnoid hemorrhage; NS means can not be analyzed.

higher proportion of a diffuse bleeding pattern on imaging studies (e.g., nPMN-SAH and intraventricular hemorrhage) $(p=0.002$ and $p<0.001$, resp., Table 3$)$.

3.3. Outcome. A total of 21 patients (12 PMN-SAH and 9 nPMN-SAH) were lost to follow-up after discharge. The mRS and GOS were used to evaluate the outcomes for all patients. We found that patients with PMN-SAH had a better clinical outcome at 3 months after ictus with respect to both mRS $(p<0.001)$ and GOS $(p=0.003)$ (Table 4). Though there was no significant difference of GOS between the two groups at 1-year follow-up ( $p=0.09$ ), the nPMN-SAH group had a trend towards a higher incidence of mild disability $(\mathrm{mRS}=2$ or $3, p=0.006$, Table 4 ).

3.4. BVR Pattern. The venous phase images on DSA for 3 NASAH patients were unavailable due to technical reasons. The distribution of BVR subtypes of three groups are listed 
TABLE 2: The clinical characteristics of nonaneurysmal subarachnoid hemorrhage.

\begin{tabular}{|c|c|c|c|c|}
\hline & All NASAH $(n=137)$ & PMN-SAH $(n=82)$ & nPMN-SAH $(n=55)$ & $p$ value \\
\hline LOS, mean (range) & $8.5 \pm 8.5(2-75)$ & $7.7 \pm 4.0(2-16)$ & $12.9 \pm 11.9(2-75)$ & 0.001 \\
\hline \multicolumn{5}{|l|}{ GCS } \\
\hline Mild (13-15) & $132(97.0 \%)$ & $82(100 \%)$ & $50(90.9 \%)$ & 0.009 \\
\hline Middle (9-12) & $1(0.7 \%)$ & 0 & $1(1.8 \%)$ & \\
\hline Severe (3-8) & $4(2.3 \%)$ & 0 & $4(7.3 \%)$ & \\
\hline \multicolumn{5}{|l|}{$\mathrm{H}$-H grade } \\
\hline Good (I-II) & $132(96.4 \%)$ & $81(98.8 \%)$ & $51(92.7 \%)$ & 0.157 \\
\hline Poor (III-IV) & $5(3.6 \%)$ & $1(1.2 \%)$ & $4(7.3 \%)$ & \\
\hline \multicolumn{5}{|l|}{$\mathrm{mFS}$} \\
\hline $0-1$ & $78(56.9 \%)$ & $70(85.4 \%)$ & $8(14.5 \%)$ & $<0.001$ \\
\hline $2-4$ & $59(43.1 \%)$ & $12(14.6 \%)$ & $47(85.5 \%)$ & \\
\hline \multicolumn{5}{|l|}{ Complication } \\
\hline Hydrocephalus & $14(10.2 \%)$ & $3(3.7 \%)$ & $11(20.0 \%)$ & 0.001 \\
\hline Cerebral vasospasm & $7(5.1 \%)$ & $1(1.2 \%)$ & $6(10.9 \%)$ & 0.017 \\
\hline Rebleeding & $1(0.7 \%)$ & 0 & $1(1.8 \%)$ & 0.401 \\
\hline Pulmonary infections & $3(2.2 \%)$ & $1(1.2 \%)$ & $2(3.6 \%)$ & 0.564 \\
\hline
\end{tabular}

NASAH: nonaneurysmal subarachnoid hemorrhage, PMN-SAH: perimesencephalic nonaneurysmal subarachnoid hemorrhage, nPMN-SAH: nonperimesencephalic nonaneurysmal subarachnoid hemorrhage, GCS: Glasgow coma scale, H-H grade: Hunt-Hess grade, mFS: modified Fisher Scale; LOS: length of hospital stay.

TABLE 3: Clinical characteristics of hydrocephalus patients in NASAH group.

\begin{tabular}{|c|c|c|c|}
\hline & No hydrocephalus $(n=123)$ & Hydrocephalus $(n=14)$ & $p$ \\
\hline \multicolumn{4}{|l|}{ Demography } \\
\hline Age & $56.4 \pm 10.0$ & $52.9 \pm 13.3$ & 0.147 \\
\hline Gender (female) & $57(46.3 \%)$ & $4(28.6)$ & 0.205 \\
\hline Smoker & $16(13.0 \%)$ & $2(14.3 \%)$ & 0.893 \\
\hline Alcohol abuse & $18(14.6 \%)$ & $3(21.4 \%)$ & 0.504 \\
\hline Hypertension & $26(21.1 \%)$ & $4(28.6 \%)$ & 0.524 \\
\hline Diabetes & $5(4.1 \%)$ & $2(14.3 \%)$ & 0.100 \\
\hline \multicolumn{4}{|l|}{ Clinical grade } \\
\hline GCS $<13$ & $0(0 \%)$ & $4(28.6 \%)$ & $<0.001$ \\
\hline Modified Fisher Scale $>1$ & $56(45.5 \%)$ & $11(78.6 \%)$ & $<0.001$ \\
\hline \multicolumn{4}{|l|}{ Bleed Pattern } \\
\hline PMN-SAH & $79(63.4 \%)$ & $3(21.4 \%)$ & 0.002 \\
\hline nPMN-SAH & $44(35.8 \%)$ & $11(78.6 \%)$ & \\
\hline Anterior circulation $^{\mathrm{a}}$ & $96(78.0 \%)$ & $12(85.7 \%)$ & 0.506 \\
\hline Posterior circulation $^{\mathrm{b}}$ & $27(21.9 \%)$ & $2(14.3 \%)$ & \\
\hline Intraventricular hemorrhage & $8(6.5 \%)$ & $10(71.4 \%)$ & $<0.001$ \\
\hline Cortical hemorrhage & $22(17.9 \%)$ & $3(21.4 \%)$ & 0.745 \\
\hline
\end{tabular}

EVD: external ventricular drain, GCS: Glasgow coma scale.

${ }^{a}$ Anterior circulation was characterized by blood mainly locating at anterior of the brain.

${ }^{\mathrm{b}}$ Posterior circulation was characterized by blood mainly locating at posterior of the brain.

in Table 5. Compared with aSAH patients, there was a significant difference of BVR distribution in both of the PMN-SAH group $(p=0.003)$ and $n P M N-S A H$ group $(p=0.021)$. However, there was no statistically significant difference of unilateral BVR development between PMNSAH and $n P M N-S A H$ groups $(p=0.950)$. To further analyze the correlation of hemorrhage type and BVR development, the bilateral BVRs were classified as four subgroups, AA,
$\mathrm{AB} / \mathrm{BB}, \mathrm{AC} / \mathrm{BC}$, and $\mathrm{CC}$, respectively. A significant statistical difference was only found between PMN-SAH and aSAH groups $(p=0.028$, Table 6$)$.

3.5. Repeated DSA Findings. To evaluate the utility of repeated DSA examination in patients with initially negative DSA findings, we compared the detection rate of a responsible intracranial lesion by the repeated DSA in both of 
TABLE 4: The prognosis of patient after charged from hospital.

\begin{tabular}{|c|c|c|c|c|}
\hline & All NASAH $(n=116)^{*}$ & PMN-SAH $(n=70)$ & nPMN-SAH $(n=46)$ & $p$ value \\
\hline \multicolumn{5}{|c|}{ mRS at 3 months } \\
\hline $0-1$ & $96(82.8 \%)$ & $66(94.3 \%)$ & $30(65.2 \%)$ & \multirow{3}{*}{$<0.001$} \\
\hline $2-3$ & $17(14.7 \%)$ & $4(5.7 \%)$ & $13(28.2 \%)$ & \\
\hline $4-6$ & $3(2.5 \%)$ & 0 & $3(6.6 \%)$ & \\
\hline \multicolumn{5}{|c|}{ GOS at 3 months } \\
\hline 5 & $110(94.8 \%)$ & $70(100 \%)$ & $40(87.0 \%)$ & \multirow{3}{*}{0.003} \\
\hline 4 & $3(3.1 \%)$ & 0 & $3(6.5 \%)$ & \\
\hline $1-3$ & $3(3.1 \%)$ & 0 & $3(6.5 \%)$ & \\
\hline \multicolumn{5}{|c|}{$\mathrm{mRS}$ at 1 year } \\
\hline $0-1$ & $78(86.7 \%)$ & $46(95.8 \%)$ & $32(76.2 \%)$ & \multirow{3}{*}{0.006} \\
\hline $2-3$ & $10(11.1 \%)$ & $2(4.2 \%)$ & $8(19.0 \%)$ & \\
\hline $4-6$ & $2(2.2 \%)$ & 0 & $2(4.8 \%)$ & \\
\hline \multicolumn{5}{|c|}{ GOS at 1 year } \\
\hline 5 & $87(96.7 \%)$ & $48(100 \%)$ & $39(92.8 \%)$ & \multirow{3}{*}{0.09} \\
\hline 4 & $1(1.1 \%)$ & 0 & $1(2.4 \%)$ & \\
\hline $1-3$ & $2(2.2 \%)$ & 0 & $2(4.8 \%)$ & \\
\hline
\end{tabular}

NASAH: nonaneurysmal subarachnoid hemorrhage; PMN-SAH: perimesencephalic nonaneurysmal subarachnoid hemorrhage; nPMN-SAH: nonperimesencephalic nonaneurysmal subarachnoid hemorrhage; GOS: Glasgow outcome scale, mRS: modified Rankin Scale.

*21 patients (12 perimesencephalic and 9 nonperimesencephalic patients) were lost to follow-up after discharge.

TABLE 5: Type of BVR in nonaneurysmal subarachnoid hemorrhage and aneurysmal subarachnoid hemorrhage patients.

\begin{tabular}{|c|c|c|c|c|c|c|}
\hline BVR type & PMN-SAH & nPMN-SAH & aSAH & $p 1$ & $p 2$ & $p 3$ \\
\hline Unilateral & $n=160$ & $n=100$ & $n=266$ & 0.003 & 0.021 & 0.950 \\
\hline A & $61(38.1 \%)$ & $39(39.0 \%)$ & $147(55.3 \%)$ & & & \\
\hline B & $48(30.0 \%)$ & $31(31.0 \%)$ & $61(22.9 \%)$ & & & \\
\hline $\mathrm{C}$ & $51(31.9 \%)$ & $30(30.0 \%)$ & $58(22.8 \%)$ & & & \\
\hline Bilateral & $n=80$ & $n=50$ & $n=133$ & 0.028 & 0.066 & 0.882 \\
\hline $\mathrm{AA}$ & $15(18.8 \%)$ & $9(18.0 \%)$ & $50(37.6 \%)$ & & & \\
\hline $\mathrm{AB}, \mathrm{BB}$ & $26(32.5 \%)$ & $16(32.0 \%)$ & $38(28.6 \%)$ & & & \\
\hline $\mathrm{AC}, \mathrm{BC}$ & $26(32.5 \%)$ & $19(38.0 \%)$ & $32(24.1 \%)$ & & & \\
\hline $\mathrm{CC}$ & $13(16.2 \%)$ & $6(12.0 \%)$ & $13(9.8 \%)$ & & & \\
\hline
\end{tabular}

BVR: basal vein of Rosenthal; PMN-SAH: perimesencephalic nonaneurysmal subarachnoid hemorrhage; nPMN-SAH: nonperimesencephalic nonaneurysmal subarachnoid hemorrhage.

$p 1$ : statistical analysis was proceeded between PMN-SAH and aSAH group.

p2: statistical analysis was proceeded nPMN-SAH and aSAH group.

p3: statistical analysis was proceeded PMN-SAH and nPMN-SAH group.

the subgroups and also included data from related literature for analysis. A total of 60 patients underwent a repeated DSA examination in our series. On repeat evaluation, 4 of 31 patients with nPMN-SAH were diagnosed with a responsible intracranial aneurysm, resulting in a detection rate of $12.9 \%$ on repeat DSA examination; however none of the PMNSAH patients had positive findings on their repeated DSA (Table 7). According to the data from recent studies, the detection rate of a responsible intracranial aneurysm by repeated DSA was $12.5 \%$ in the nPMN-SAH subgroup and $1.2 \%$ in the PMN-SAH subgroup (Table 7 ).

\section{Discussion}

SAH patients with negative initial DSA findings, usually defined as NASAH, can be a management challenge as the optimal management scheme for these patients remains controversial. The present study analyzes the data of SAH patients with negative initial DSA findings from our center. We divided those patients into the two subgroups of PMN$\mathrm{SAH}$ and nPMN-SAH. The development of BVR type in each group was compared with an aSAH control group to help elucidate the source of bleeding. The necessity of repeated DSA examinations was evaluated in each group by calculating the positive finding rate from second angiogram.

It is wildly accepted that NASAH patients have a more favorable clinical course and a lower incidence of complications when compared to those with aSAH [34]. The subgroup of PMN-SAH was first described by van Gijn et al. [35] as a benign entity, characterized by the distribution of the subarachnoid hemorrhage mainly or only in the perimesencephalic cisterns. However, the nPMN-SAH group has 


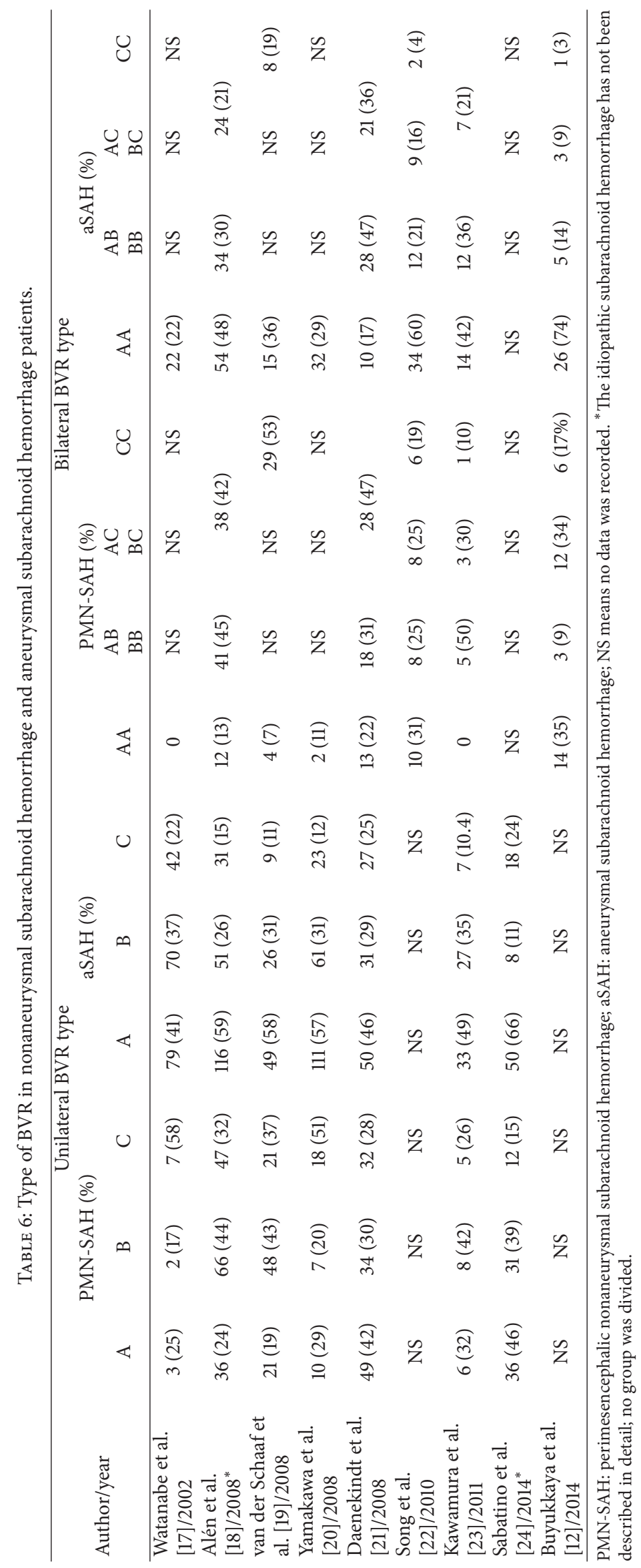


TABLE 7: Diagnostic yield of a repeated DSA investigation.

\begin{tabular}{|c|c|c|c|c|c|c|c|}
\hline Author/year & Study type & PMN-SAH & Positive & Misdiagnose rate & nPMN-SAH & Positive & Misdiagnose rate \\
\hline $\begin{array}{l}\text { Topcuoglu et al. } \\
{[10] / 2003}\end{array}$ & Retrospective & 31 & 0 & 0 & 36 & 3 & $8.3 \%$ \\
\hline $\begin{array}{l}\text { Jung et al. } \\
{[25] / 2006}\end{array}$ & Retrospective & 65 & 1 & $1.5 \%$ & 37 & 17 & $45.9 \%$ \\
\hline $\begin{array}{l}\text { Huttner et al. } \\
{[11] / 2006}\end{array}$ & Prospective & 38 & 0 & 0 & NS & NS & NS \\
\hline $\begin{array}{l}\text { Little et al. } \\
{[26] / 2007}\end{array}$ & Retrospective & 23 & 1 & $4.3 \%$ & 59 & 5 & $8.5 \%$ \\
\hline $\begin{array}{l}\text { Gupta et al. } \\
\text { [3]/2009 }\end{array}$ & Retrospective & 18 & 0 & 0 & 43 & 2 & $4.7 \%$ \\
\hline $\begin{array}{l}\text { Carvi y Nievas and } \\
\text { Archavlis } \\
{[27] / 2009}\end{array}$ & Retrospective & 8 & 0 & 0 & 3 & 1 & $33.3 \%$ \\
\hline Agid et al. [9]/2010 & Retrospective & 28 & 0 & 0 & 28 & 4 & $14.3 \%$ \\
\hline $\begin{array}{l}\text { Fontanella et al. } \\
\text { [28]/2011 }\end{array}$ & Retrospective & 23 & 0 & 0 & 72 & 9 & $12.5 \%$ \\
\hline $\begin{array}{l}\text { Maslehaty et al. } \\
\text { [29]/2011 }\end{array}$ & Retrospective & 34 & 1 & $2.9 \%$ & 120 & 13 & $10.8 \%$ \\
\hline $\begin{array}{l}\text { Kelliny et al. } \\
{[30] / 2011}\end{array}$ & Retrospective & 35 & 0 & 0 & 37 & 6 & $16.2 \%$ \\
\hline $\begin{array}{l}\text { Delgado Almandoz } \\
\text { et al. [31]/2012 }\end{array}$ & Prospective & 29 & 1 & $3.4 \%$ & 39 & 2 & $5.1 \%$ \\
\hline Lin et al. [32]/2012 & Retrospective & 27 & 0 & 0 & 41 & 2 & $4.9 \%$ \\
\hline DW et al. [8]/2012 & Retrospective & 6 & 0 & 0 & 12 & 2 & $16.7 \%$ \\
\hline Present study & Retrospective & 31 & 0 & 0 & 29 & 4 & $13.8 \%$ \\
\hline \multicolumn{2}{|l|}{ Total } & 365 & 4 & $1.1 \%$ & 556 & 70 & $12.6 \%$ \\
\hline
\end{tabular}

PMN-SAH: perimesencephalic nonaneurysmal subarachnoid hemorrhage; nPMN-SAH: nonperimesencephalic nonaneurysmal subarachnoid hemorrhage; NS means no data was recorded.

a more diffuse distribution of the subarachnoid hemorrhage that is more similar to aSAH [15]. A diagnosis of nPMN$\mathrm{SAH}$ is associated with higher rate of complications, such as hydrocephalus, cerebral vasospasm, and cerebral infarction [5]. Hydrocephalus is one of the most common complications of NASAH, normally identified by symptoms of elevated intracranial pressure (progressive headache, altered sensorium) or findings on head CT [36]. The incidence of hydrocephalus from the present cohort was $14.3 \%$, which is in agreement with previous reports [34]. We also found that, in the nonperimesencephalic pattern, the presence of intraventricular hemorrhage or poorer clinical grade on presentation (GCS $<13$ and modified Fisher Scale $>2$ ) were associated with the development of hydrocephalus.

Interestingly, despite the repeated imaging studies that have been performed on NASAH patients, the etiology of bleeding remains obscure. The potential pathogenesis may include a venous system variant [20], capillary abnormality, intracranial basilar dissection, ruptured perforating artery [37], cavernous malformation [38], or capillary telangiectasia [15]. Although both arterial and venous origins for the SAH have been proposed, most of the studies favor a venous source of PMN-SAH [39]. It was first hypothesized by Watanabe et al. [17] that a large portion of the BVRs in PMN-SAH patients vary from a normal configuration with drainage into dural sinuses instead of the vein of Galen. Similarly, van der Schaaf et al. [19] reported that primitive venous drainage was more common in patients with PMN$\mathrm{SAH}$ and that the venous drainage variation was ipsilateral to the side of bleeding. Most of the subsequent studies supported the theory that the drainage into the deep venous drain system (the vein of Rosenthal variant) was related to the PMN-SAH [12, 18-20, 22-24]. However, a study from Daenekindt et al. [21] suggested otherwise (Table 6), but this may be explained by its relatively small patient series, short follow-up period, or differences in diagnostic criteria. Similar to prior publications, our present study demonstrated a significant difference in BVR anatomy between the PMN$\mathrm{SAH}$ and aSAH groups, regardless of unilateral or bilateral BVR type $(p=0.003,0.028)$ and that the primitive venous configuration was ipsilateral to the source of the bleeding. However, it remains unclear exactly how the venous drainage variation contributes to PMN-SAH.

However, unlike with the PMN-SAH group, the relationship between venous drainage and nPMN-SAH was rarely described previously. It is generally hypothesized that the nPMN pattern may have resulted from an arterial source to account for its relatively "malignant" clinical presentation and extensive blood distribution into the cisterns and parenchyma. Consequently, this pattern of $\mathrm{SAH}$ is also 
referred to as aneurysm-like SAH. Interestingly, our study revealed that there was also a significant difference in the unilateral BVR type between the nPMN-SAH and aSAH groups $(p=0.021)$. However, for bilateral venous drainage, there was no significant difference between the two groups ( $p=0.066$ ). The inconsistent findings between the unilateral and bilateral BVR types could be explained by a contribution of true aSAH that were misclassified as nPMN-SAH because of an initial negative DSA. Although a higher portion of nPMN-SAH with negative initial DSA findings were eventually confirmed to have resulted from ruptured intracranial aneurysms, compared to PMN-SAH, most sources of nPMNSAH remain idiopathic. Therefore, we conclude that if a ruptured intracranial aneurysm can be absolutely excluded, the bleeding source of a true nPMN-SAH may be similar to that found in PMN-SAH.

Another main concern in the management of NASAH is to evaluate the necessity of repeated DSA examinations for each individual. While DSA is currently the standard method to diagnose an intracranial aneurysm for patients suffering from SAH, there are risks to the procedure. Some studies have shown that catheter angiography has up to a $2.6 \%$ risk of permanent neurologic complications in NASAH [40]. Recently, numerous reports have proposed the use of noninvasive techniques to diagnose an intracranial aneurysm, such as CTA and magnetic resonance angiography (MRA) [41, 42]. The accuracy of the DSA result can be affected by numerous factors: the resolution of the DSA device, the quality of the acquired scan, the 3D reconstruction capabilities, the interval between the onset of the symptoms and the examination, and the experience of the technician. Furthermore, a small or dissecting aneurysm, hemorrhage or vasospasm concealing the aneurysm, or technical deficiencies can lead to a falsenegative result upon the initial examination [43]. Even though improvements in imaging technology have decreased the incidence of misdiagnosis, there are still nearly $15 \%$ of SAH patients who have negative findings upon their initial DSA examination [43, 44]. Since missing an aneurysmal source on DSA would expose patients to the extensive morbidity and mortality of rebleed, most practitioners carry out repeat DSA exams on SAH patients with a negative initial DSA. Consequently, it would be of value to select the specific individuals who would benefit from repeated DSA exams.

According to our present study, a total of 60 patients underwent a repeat DSA examination. Four of 31 patients with nPMN-SAH were ultimately diagnosed with a ruptured intracranial aneurysm, resulting in a detection rate of $12.9 \%$ for a repeat DSA. However none of the PMN-SAH patients in our study had positive findings on their repeat DSA. To further address this finding, a thorough review of data from past studies was evaluated. There are 13 studies in the past 15 years that discuss a positive finding on repeat DSA exams in SAH patients with negative initial DSA findings. For the pooled data of PMN-SAH patients, a repeat DSA detected a culprit aneurysm in only 4 of 151 patients $[2,3,8,27,28,30,32]$ (Table 7) $[25,26,29,31]$. Combined with our data, the overall misdiagnosis rate is only $1.1 \%$, which is likely lower than the risk of the DSA procedure itself. We therefore propose that it may be acceptable to follow up PMN-SAH patients by noninvasive image studies rather than DSA. Some authors go further, even raising the possibility that it is reasonable to manage PMN-SAH patients completely with noninvasive cerebral vascular imaging. The preference at our institution is that unless the initial CT scan with high quality vascular imaging is completed within several hours after the onset of symptoms, all PMN-SAH patients need at least one DSA to exclude a ruptured intracranial aneurysm. However, for centers without available neurointerventionalists, the DSA can be delayed because PMN-SAH is usually associated with a benign clinical course and good prognosis. Unlike the extremely low rate of aneurysm detection in PMN-SAH patients, our data revealed that $12.6 \%$ of nPMN-SAH patients were ultimately found to have a responsible aneurysm on the second DSA. In previous studies, the misdiagnosis rate of the initial DSA in nPMN-SAH patients varied greatly (misdiagnose rate from $4.7 \%$ to $45.9 \%$ ), which is likely due to several factors, as previously mentioned. After pooling all the published studies, the overall misdiagnose rate was $12.5 \%$ ( 66 out of 527, Table 7), which is similar to the findings at our institution. Therefore, we strongly recommend a repeat DSA examination for patients with $\mathrm{PPMN}-\mathrm{SAH}$ who had negative initial findings. Additionally, it is advisable to use 3D image acquisition for both internal carotid arteries and the vertebral arteries in the initial and repeat DSA to optimize the chances of finding small lesions. Since the aneurysms discovered on repeat imaging tend to be miniature in size and are found at the bifurcation of small perforating arteries, we also suggest consultation with an experienced neuroradiological or/and neurointervention physician to establish the diagnosis.

\section{Conclusion}

Managing SAH patients with negative initial DSA findings can be challenging. Based on the results of our present study and a review of the pertinent literature, the PMN-SAH subgroup usually has a benign clinical course and a repeat DSA very seldom reveals a ruptured intracranial aneurysm. More importantly, nPMN-SAH patients are associated with higher complication rate and incidence of a ruptured aneurysm. Therefore, we strongly recommend a repeat DSA in patients with nPMN-SAH pattern on initial imaging.

\section{Conflicts of Interest}

The authors report no conflicts of interest concerning the materials or methods used in this study or findings specified in this paper.

\section{Authors' Contributions}

Drs Liang Xu and Yuanjian Fang contributed equally to this work.

\section{Acknowledgments}

The authors thank Rudi Scharnweber for revising the manuscript. This study was funded by the National Natural Science Foundation of China (no. 81671131 to Jing Xu). 


\section{References}

[1] B. Lucke-Wold, A. Logsdon, B. Manoranjan et al., "Aneurysmal subarachnoid hemorrhage and neuroinflammation: a comprehensive review," International Journal of Molecular Sciences, vol. 17, no. 4, article 497, 2016.

[2] Y. Hasegawa, H. Suzuki, K. Uekawa, T. Kawano, and S. KimMitsuyama, "Characteristics of cerebrovascular injury in the hyperacute phase after induced severe subarachnoid hemorrhage," Translational Stroke Research, vol. 6, no. 6, pp. 458-466, 2015.

[3] S. K. Gupta, R. Gupta, V. K. Khosla et al., "Nonaneurysmal nonperimesencephalic subarachnoid hemorrhage: is it a benign entity?" Surgical Neurology, vol. 71, no. 5, pp. 566-572, 2009.

[4] G. J. Rinkel, E. F. Wijdicks, M. Vermeulen et al., "Nonaneurysmal perimesencephalic subarachnoid hemorrhage: CT and MR patterns that differ from aneurysmal rupture," AJNR American Journal of Neuroradiology, vol. 12, no. 5, pp. 829-834, 1991.

[5] Y. Kong, J. H. Zhang, and X. Qin, "Perimesencephalic subarachnoid hemorrhage: risk factors, clinical presentations, and outcome," Acta Neurochirurgica Supplement, vol. 110, part 1, pp. 197-201, 2011.

[6] C. P. Du and K. F. Ke, "The clinical analysis of nonaneurysmal subarachnoid hemorrhage," Zhonghua Nei Ke Za Zhi, vol. 50, pp. 408-410, 2011.

[7] L. L. Herrmann and J. M. Zabramski, "Nonaneurysmal subarachnoid hemorrhage: a review of clinical course and outcome in two hemorrhage patterns," The Journal of Neuroscience Nursing : Journal of The American Association of Neuroscience Nurses, vol. 39, no. 3, pp. 135-142, 2007.

[8] D. W. Yu, Y. J. Jung, B. Y. Choi, and C. H. Chang, "Subarachnoid hemorrhage with negative baseline digital subtraction angiography: is repeat digital subtraction angiography necessary?" Journal of Cerebrovascular and Endovascular Neurosurgery, vol. 14, no. 3, pp. 210-215, 2012.

[9] R. Agid, T. Andersson, H. Almqvist et al., "Negative CT angiography findings in patients with spontaneous subarachnoid hemorrhage: when is digital subtraction angiography still needed?" American Journal of Neuroradiology, vol. 31, no. 4, pp. 696-705, 2010.

[10] M. A. Topcuoglu, C. S. Ogilvy, B. S. Carter, F. S. Buonanno, W. J. Koroshetz, and A. B. Singhal, "Subarachnoid hemorrhage without evident cause on initial angiography studies: diagnostic yield of subsequent angiography and other neuroimaging tests," Journal of Neurosurgery, vol. 98, no. 6, pp. 1235-1240, 2003.

[11] H. B. Huttner, M. Hartmann, M. Köhrmann et al., "Repeated digital substraction angiography after perimesencephalic subarachnoid hemorrhage?" Journal of Neuroradiology, vol. 33, no. 2, pp. 87-89, 2006.

[12] R. Buyukkaya, N. Yildirim, H. Cebeci et al., "The relationship between perimesencephalic subarachnoid hemorrhage and deep venous system drainage pattern and calibrations," Clinical Imaging, vol. 38, no. 3, pp. 226-230, 2014.

[13] S. A. Dupont, G. Lanzino, E. F. M. Wijdicks, and A. A. Rabinstein, "The use of clinical and routine imaging data to differentiate between aneurysmal and nonaneurysmal subarachnoid hemorrhage prior to angiography: clinical article," Journal of Neurosurgery, vol. 113, no. 4, pp. 790-794, 2010.

[14] H. Suzuki, M. Shiba, Y. Nakatsuka, F. Nakano, and H. Nishikawa, "Higher cerebrospinal fluid $\mathrm{pH}$ may contribute to the development of delayed cerebral ischemia after aneurysmal subarachnoid hemorrhage," Translational Stroke Research, vol. 8, no. 2, pp. 165-173, 2017.

[15] Y. W. Kim, M. F. Lawson, and B. L. Hoh, "Nonaneurysmal subarachnoid hemorrhage: an update," Current Atherosclerosis Reports, vol. 14, no. 4, pp. 328-334, 2012.

[16] P. Kang, A. Raya, G. J. Zipfel, and R. Dhar, "Factors associated with acute and chronic hydrocephalus in nonaneurysmal subarachnoid hemorrhage," Neurocritical Care, vol. 24, no. 1, pp. 104-109, 2016.

[17] A. Watanabe, K. Hirano, M. Kamada et al., "Perimesencephalic nonaneurysmal subarachnoid haemorrhage and variations in the veins," Neuroradiology, vol. 44, no. 4, pp. 319-325, 2002.

[18] J. F. Alén, A. Lagares, J. Campollo et al., "Idiopathic subarachnoid hemorrhage and venous drainage: are they related?" Neurosurgery, vol. 63, no. 6, pp. 1106-1112, 2008.

[19] I. C. van der Schaaf, B. K. Velthuis, A. Gouw, and G. J. E. Rinkel, "Venous drainage in perimesencephalic hemorrhage," Stroke, vol. 35, no. 7, pp. 1614-1618, 2004.

[20] H. Yamakawa, N. Ohe, H. Yano, S. Yoshimura, and T. Iwama, "Venous drainage patterns in perimesencephalic nonaneurysmal subarachnoid hemorrhage," Clinical Neurology and Neurosurgery, vol. 110, no. 6, pp. 587-591, 2008.

[21] T. Daenekindt, G. Wilms, V. Thijs, P. Demaerel, and F. Van Calenbergh, "Variants of the basal vein of Rosenthal and perimesencephalic nonaneurysmal hemorrhage," Surgical Neurology, vol. 69, no. 5, pp. 526-529, 2008.

[22] J. H. Song, J. Y. Yeon, K. H. Kim, P. Jeon, J. S. Kim, and S.-C. Hong, "Angiographic analysis of venous drainage and a variant basal vein of Rosenthal in spontaneous idiopathic subarachnoid hemorrhage," Journal of Clinical Neuroscience, vol. 17, no. 11, pp. 1386-1390, 2010.

[23] Y. Kawamura, O. Narumi, M. Chin, and S. Yamagata, "Variant deep cerebral venous drainage in idiopathic subarachnoid hemorrhage," Neurologia Medico-Chirurgica, vol. 51, no. 2, pp. 97-100, 2011

[24] G. Sabatino, G. M. Della Pepa, A. Scerrati et al., "Anatomical variants of the basal vein of Rosenthal: prevalence in idiopathic subarachnoid hemorrhage," Acta Neurochirurgica, vol. 156, no. 1, pp. 45-51, 2014.

[25] J. Y. Jung, Y. B. Kim, J. W. Lee, S. K. Huh, and K. C. Lee, "Spontaneous subarachnoid haemorrhage with negative initial angiography: a review of 143 cases," Journal of Clinical Neuroscience, vol. 13, no. 10, pp. 1011-1017, 2006.

[26] A. S. Little, M. Garrett, R. Germain et al., "Evaluation of patients with spontaneous subarachnoid hemorrhage and negative angiography," Neurosurgery, vol. 61, no. 6, pp. 1139-1151, 2007.

[27] M. N. Carvi y Nievas and E. Archavlis, "Atypical causes of nontraumatic intracranial subarachnoid hemorrhage," Clinical Neurology and Neurosurgery, vol. 111, no. 4, pp. 354-358, 2009.

[28] M. Fontanella, I. Rainero, P. P. Panciani et al., "Subarachnoid hemorrhage and negative angiography: clinical course and long-term follow-up," Neurosurgical Review, vol. 34, no. 4, pp. 477-484, 2011.

[29] H. Maslehaty, A. K. Petridis, H. Barth, and H. M. Mehdorn, "Diagnostic value of magnetic resonance imaging in perimesencephalic and nonperimesencephalic subarachnoid hemorrhage of unknown origin: clinical article," Journal of Neurosurgery, vol. 114, no. 4, pp. 1003-1007, 2011.

[30] M. Kelliny, P. Maeder, S. Binaghi, M. Levivier, L. Regli, and R. Meuli, "Cerebral aneurysm exclusion by CT angiography based 
on subarachnoid hemorrhage pattern: a retrospective study," BMC Neurology, vol. 11, article 8, 2011.

[31] J. E. Delgado Almandoz, B. D. Jagadeesan, D. Refai et al., "Diagnostic yield of repeat catheter angiography in patients with catheter and computed tomography angiography negative subarachnoid hemorrhage," Neurosurgery, vol. 70, no. 5, pp. 1135-1142, 2012.

[32] N. Lin, G. Zenonos, A. H. Kim et al., "Angiogram-negative subarachnoid hemorrhage: relationship between bleeding pattern and clinical outcome," Neurocritical Care, vol. 16, no. 3, pp. 389398, 2012.

[33] C. M. Brown, C. D. Bushnell, G. P. Samsa, L. B. Goldstein, and C. A. Colton, "Chronic systemic immune dysfunction in africanamericans with small vessel-type ischemic stroke," Translational Stroke Research, vol. 6, no. 6, pp. 430-436, 2015.

[34] A. Kapadia, T. A. Schweizer, J. Spears, M. Cusimano, and R. L. Macdonald, "Nonaneurysmal perimesencephalic subarachnoid hemorrhage: diagnosis, pathophysiology, clinical characteristics, and long-term outcome," World Neurosurgery, vol. 82, no. 6, pp. 1131-1143, 2014.

[35] J. van Gijn, K. J. van Dongen, M. Vermeulen, and A. Hijdra, "Perimesencephalic hemorrhage: a nonaneurysmal and benign form of subarachnoid hemorrhage," Neurology, vol. 35, no. 4, pp. 493-497, 1985.

[36] F. Schlunk and S. M. Greenberg, "The pathophysiology of intracerebral hemorrhage formation and expansion," Translational Stroke Research, vol. 6, no. 4, pp. 257-263, 2015.

[37] M. G. Lansberg, "Concurrent presentation of perimesencephalic subarachnoid hemorrhage and ischemic stroke," Journal of Stroke and Cerebrovascular Diseases, vol. 17, no. 4, pp. 248250, 2008.

[38] A. Kurosu, K. Suzukawa, M. Amo, N. Horinaka, and H. Arai, "Perimesencephalic non-aneurysmal subarachnoid hemorrhage caused by cavernous sinus thrombosis-case report," Neurologia Medico-Chirurgica, vol. 47, no. 6, pp. 258-260, 2007.

[39] J. P. Cruz, D. Sarma, and L. Noel de Tilly, "Perimesencephalic subarachnoid hemorrhage: when to stop imaging?" Emergency Radiology, vol. 18, no. 3, pp. 197-202, 2011.

[40] Y. M. Ruigrok, G. J. E. Rinkel, E. Buskens, B. K. Velthuis, and J. Van Gijn, "Perimesencephalic hemorrhage and CT angiography: a decision analysis," Stroke, vol. 31, no. 12, pp. 29762983, 2000.

[41] M. T. Karamessini, G. C. Kagadis, T. Petsas et al., "CT angiography with three-dimensional techniques for the early diagnosis of intracranial aneurysms. Comparison with intra-arterial DSA and the surgical findings," European Journal of Radiology, vol. 49, no. 3, pp. 212-223, 2004.

[42] K. W. Muir and I. M. Macrae, "Neuroimaging as a selection tool and endpoint in clinical and pre-clinical trials," Translational Stroke Research, vol. 7, no. 5, pp. 368-377, 2016.

[43] A. Hochmuth, U. Spetzger, and M. Schumacher, "Comparison of three-dimensional rotational angiography with digital subtraction angiography in the assessment of ruptured cerebral aneurysms," AJNR American Journal of Neuroradiology, vol. 23, no. 7, pp. 1199-1205, 2002.

[44] H. Ishihara, S. Kato, T. Akimura, E. Suehiro, T. Oku, and M. Suzuki, "Angiogram-negative subarachnoid hemorrhage in the era of three dimensional rotational angiography," Journal of Clinical Neuroscience, vol. 14, no. 3, pp. 252-255, 2007. 


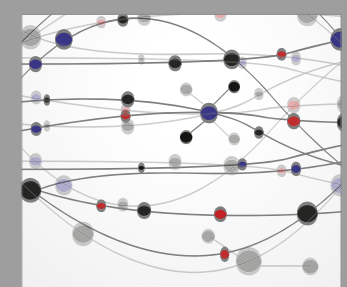

The Scientific World Journal
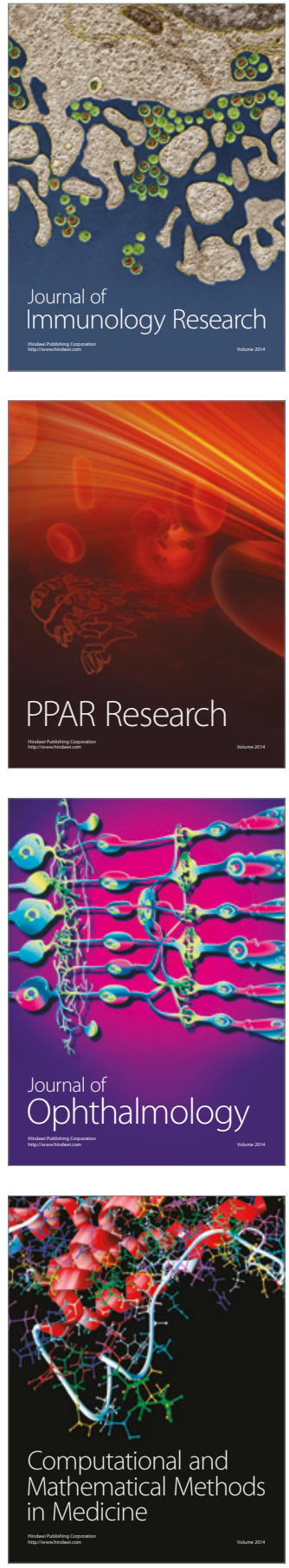

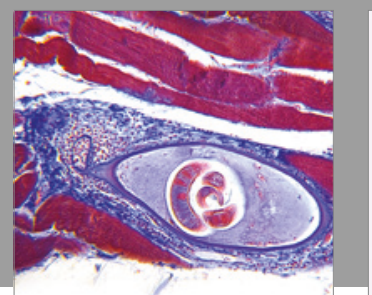

Gastroenterology Research and Practice
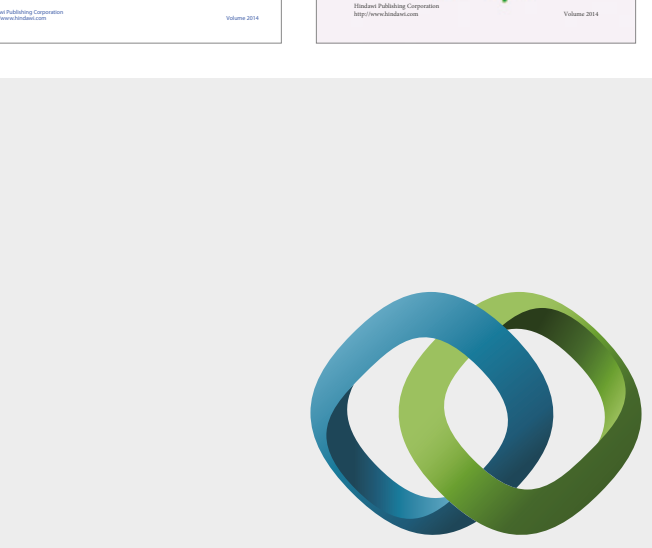

\section{Hindawi}

Submit your manuscripts at

https://www.hindawi.com
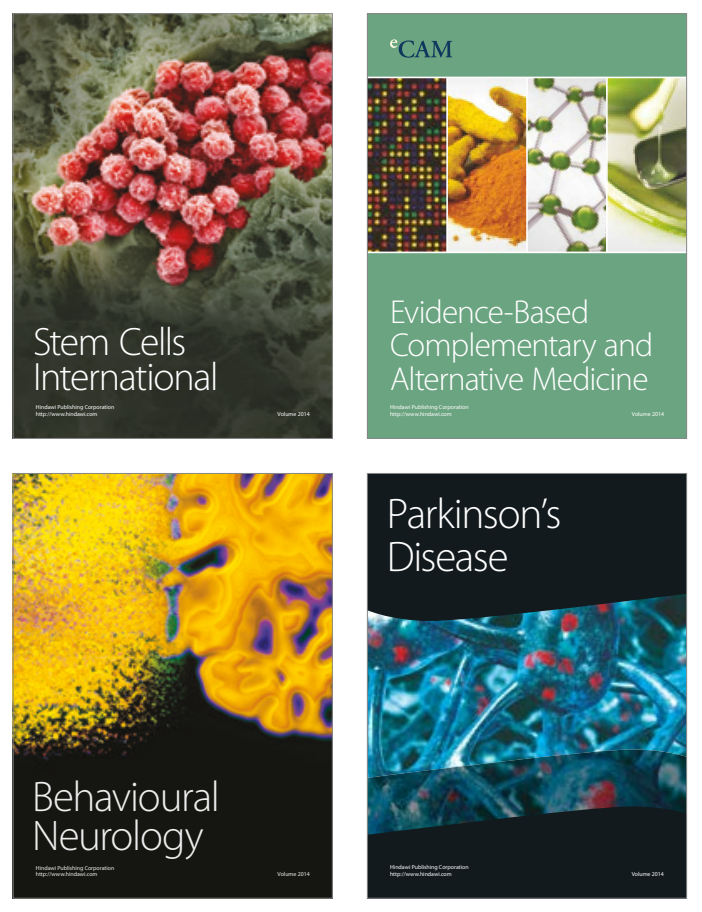
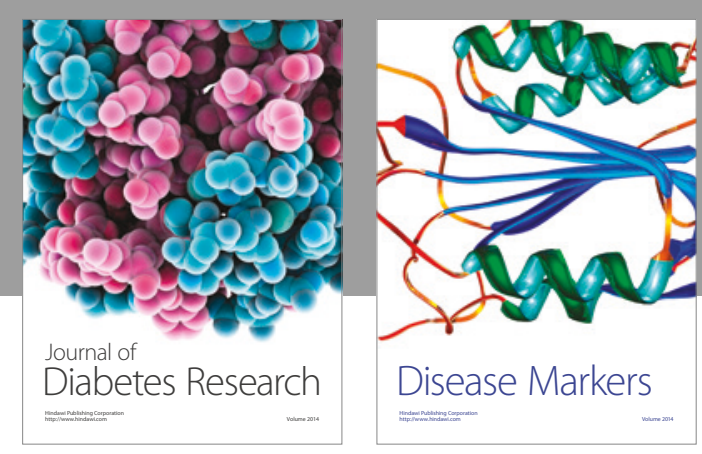

Disease Markers
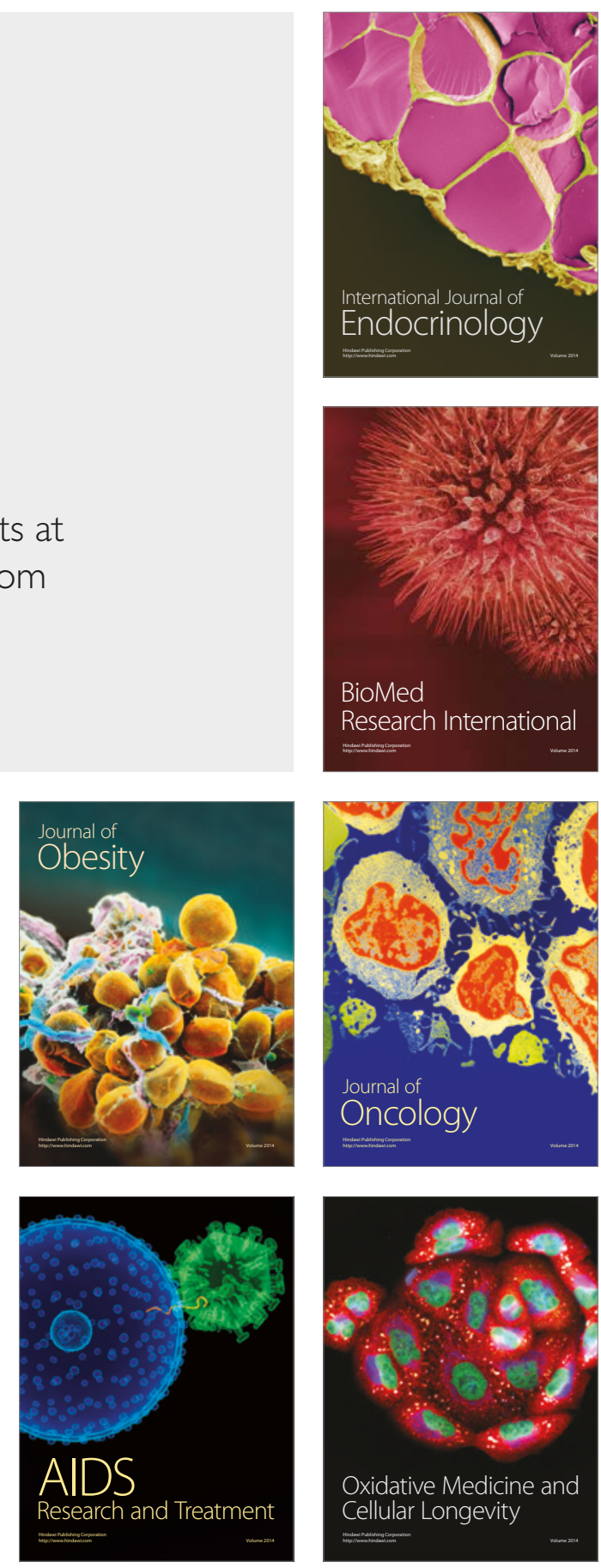